\title{
Does convective core overshooting depend on stellar mass?
}

\section{Tests using double-lined eclipsing binaries $\star$}

\author{
A. Claret \\ Instituto de Astrofísica de Andalucía, CSIC, Apartado 3004, 18080 Granada, Spain
}

e-mail: claret@iaa.es

Received 6 June 2007 / Accepted 18 September 2007

ABSTRACT

\begin{abstract}
Aims. We have selected 13 double-line eclipsing binary systems (DLEBS), strategically positioned in the HR diagram, to infer the mass dependence of the core overshooting parameter $\alpha_{\mathrm{ov}}$.

Methods. In order to compare the data from these DLEBS with the theoretical predictions we computed four grids of evolutionary stellar models with variable amounts of core overshooting $\left(\alpha_{\mathrm{ov}}=0.0,0.2,0.4\right.$, and 0.6$)$ in addition to those previously computed. In some particular cases specific models were computed for the precise observed masses. This procedure avoids interpolations and extrapolations, and only considers chemical compositions within the limits of the usual primordial helium abundance and enrichment law.

Results. We used the ratio TR $\equiv T_{\text {eff } 2} / T_{\text {eff1 } 1}$ instead of the effective temperatures themselves as we consider it a much better constrained parameter. This is because it is directly derived from the analysis of light curves and is independent of calibrations, distances, and model atmospheres. In contrast to previous studies on the subject, we have found that standard models or models with moderate core overshooting $\left(\alpha_{\mathrm{ov}} \approx 0.2\right)$ are able to match the absolute dimensions of key DLEBS such as V380 Cyg or V453 Cyg. The resulting $\log M-\alpha_{\mathrm{ov}}$ diagram indicates that the dependence of $\alpha_{\mathrm{ov}}$ on mass is more uncertain and less pronounced that previously established. This result is also consistent with the analysis of colour-magnitude diagrams for clusters and with previous comparisons of stellar models with larger samples of DLEBS.
\end{abstract}

Key words. stars: binaries: eclipsing - stars: evolution - stars: interiors

\section{Introduction}

There are still severe uncertainties in the theory of transport of energy by convection in stars. This directly affects the modelling of the external layers of colder stars as well as the calculation of the core properties of more massive ones. In the latter case, this limitation is particularly important when one considers core overshooting. The associated free parameters, $\alpha$ for mixinglength and $\alpha_{\mathrm{ov}}$ for core overshooting, are adjusted by comparing the theoretical models with suitable observations.

The first theoretical investigations on core overshooting were carried out by Saslaw \& Schwarzschild (1965), Shaviv $\&$ Salpeter (1973) and Maeder (1975). Since these pioneering papers the literature has reached at a consensus on the average value of $\alpha_{\mathrm{ov}}$ - the quantity which characterizes the distance that convective elements penetrate beyond the classical core in terms of pressure scale height - for more massive stars of approximately 0.25 (Schaller et al. 1992; Claret 1995). There are some alternative formulations of core overshooting which take into account, for example, the influence of the radiation pressure on the extra distance $d_{\text {ov }}$ (Pols et al. 1995). The resulting models are, however, nearly equivalent to those computed adopting $\alpha_{\mathrm{ov}} \approx 0.25$.

Given that the extra distance depends on the physical conditions at the border of the convective core, one natural question arises: does the core overshooting amount depend on these

* Figures 1-12 are only available in electronic form at http://www . aanda. org conditions and more specifically, on the core mass or on chemical composition? Straus et al. (1976) investigated extending the mixing region to try to understand the lithium depletion pattern in low-mass stars. They found that the overshooting amount in the external layers (in terms of the pressure scale height) depends on the stellar mass. Concerning the mass dependence of core overshooting, important studies were published by Xiong (1985, 1986) and Kuhfuss (1987) which indicated an abrupt decrease of the overshooting near stellar masses of 1.1-1.5 $M_{\odot}$. The advent of the new opacity tables by Rogers \& Iglesias (1992) has changed this scenario a little. Stothers \& Chin (1991, 1992), by adopting those tables, concluded that core overshooting is not necessary. However, some authors (Schaller et al. 1992; Bressan et al. 1993; Claret 1995) found that core oversooting was still needed.

The data from the double-lined eclipsing binary systems (DLEBS) are, in principle, a good tool to test the predictions of theoretical stellar models. One of the first papers where the importance of the core overshooting using DLEBS was investigated, is that by Andersen et al. A. (1990). In this paper, 8 systems with masses around $2 M_{\odot}$ were used to compare the predictions of standard models with models taking into account core overshooting. Although the mass dependence with the core overshooting parameter was not investigated, Andersen A. et al. (1990) found that non-standard models are necessary to fit the properties of those systems with masses larger than $1.5 M_{\odot}$. They also found that this result is compatible with the need to include core overshooting to fit satisfactorily the colour-magnitude 
diagram (CMD) of two clusters (IC 4651 and NGC 3680). On the other hand, Claret \& Giménez $(1991,1993)$ have shown that a moderate core overshooting improves the comparison between the theoretical apsidal motion constants and the observational data. It is important to remember that before this comparison is made, the theoretical stellar models should match the observed masses, radii, and effective temperatures of both components at the same age. However, a systematic discrepancy concerning the apsidal motion constants still remained. These authors tried to explain this by adopting variable core overshooting and comparing the calculations with data from seven eclipsing binaries at different stages of evolution (Y Cyg, QX Car, EM Car, V451 Oph, V453 Cyg, V380 Cyg and $\alpha$ Vir). Unfortunately, some of these systems, in particular V380 Cyg and $\alpha$ Vir, presented severe problems with their absolute dimensions, despite their very interesting position in the HR diagram. It was found that only with an unrealistically high value of $\alpha_{\mathrm{ov}}$, not supported by the observational evidence, was it possible to match the observed apsidal motion rates.

Later Umezu (1995), using homogeneous models, found a dependence of the size of the extra mixing region on mass: for stars with masses smaller than $5 M_{\odot}$ it was found that there was a decrease of mass fraction of the overshooting region with decreasing mass, with a rapid decrease near $1.5 M_{\odot}$. More recently Ribas et al. (2000) used eight DLEBS to infer the dependence on mass of core overshooting. However, the method adopted by these authors has been questioned, mainly in the case of V380 Cyg (Claret 2003). In this paper, we revise the cases of 13 DLEBS, strategically positioned in the HR diagram, to infer the dependence on mass (if it exists) of the core overshooting parameter. In order to do that, we computed four grids of stellar models, centred on $(X, Z)=(0.70,0.02)$, with different values of $\alpha_{\mathrm{ov}}$ of $0.0,0.2,0.4$ and 0.6. As auxiliary tools we also use the series of grids published over the last 3 years (Claret 2004, 2007). In addition, stellar models were computed specifically for precise observed masses for some systems.

\section{Stellar evolutionary models}

The stellar models were generated by the Granada stellar evolutionary code that is described in detail in Claret (2004). For all stellar models we take for the excess distance $d_{\text {over }}=\alpha_{\mathrm{ov}} H_{\mathrm{p}}$, where $H_{\mathrm{p}}$ is the pressure scale height taken at the edge of the convective core as given by Schwarzschild's criterion and $\alpha_{\mathrm{ov}}$ is a free parameter. In addition to the previously computed models (see Claret 2007, for a complete list) we computed four additional grids with variable amounts of core overshooting $\left(\alpha_{\mathrm{ov}}=\right.$ $0.0,0.2,0.4$, and 0.6). We adopted for these four grids $(X, Z)=$ $(0.70,0.02)$ which is representative of a large sample of DLEBS, as shown by Claret \& Willems (2002). For completeness and quick reference we summarize below the main characteristics of the grids:

1. Chemical composition and mass range. The models cover a large metal content $\mathrm{Z}$, the lowest being 0.002 and the highest 0.10 , a factor of 50 . These values completely cover the range of metallicities often found in DLEBS. Therefore, no extrapolations in chemical composition are needed. The models were computed adopting the primordial helium content as derived by Claret \& Willems (2002), $Y_{\mathrm{p}}=0.238 \pm 0.011$, with a mean enrichment law given by $\Delta Y / \Delta Z \approx 2.0$. As we will see in the next sections, no models with the helium content $Y$ smaller than $Y_{\mathrm{p}}$ will be considered. The mass range covered by every grid is $0.8-125 M_{\odot}$.
2. Convection. For colder models, we adopted the mixinglength theory to describe their envelopes, with $\alpha=1.68$. For models with convective cores, a variable amount of core overshooting was taken into account, as explained above.

3. Opacities. For high temperatures the code uses the set of tables of opacities provided by Iglesias \& Rogers (1996), completed by the calculations by Alexander \& Fergunson (1994) for lower temperatures.

4. Mass loss. Although mass loss is not too important here given the evolutionary status of most of the DLEBS studied, the formalism by Nieuwenhuijzen \& de Jager (1990) is taken into account for all models except for Wolf-Rayet stars (Nugis \& Lamers et al. 2000) and for red giants with masses smaller than $4 M_{\odot}$ (Reimers 1977).

5. Nuclear network. A more general network can be used for more advanced stages of evolution (47 isotopes). However, such a subroutine requires very time-consuming calculations so we adopted a less extensive network which follows the evolution of 13 isotopes: ${ }^{1} \mathrm{H},{ }^{4} \mathrm{He},{ }^{12} \mathrm{C},{ }^{13} \mathrm{C},{ }^{14} \mathrm{~N},{ }^{16} \mathrm{O},{ }^{17} \mathrm{O}$, ${ }^{18} \mathrm{O},{ }^{20} \mathrm{Ne},{ }^{22} \mathrm{Ne},{ }^{24} \mathrm{Mg},{ }^{25} \mathrm{Mg}$, and ${ }^{26} \mathrm{Mg}$. Recent measurements of the nuclear reaction ${ }^{14} \mathrm{~N}(\mathrm{p}, \gamma){ }^{15} \mathrm{O}$ (Runkle 2003; Formicola et al. 2004) are also incorporated into the code. The new rate is around $50 \%$ of the old one.

Once the models were computed, we generated suitable files to compute isochrones for every grid. For those systems which show slight deviations from the initial chemical compositions adopted in our series of grids, we computed specific models for the precise observed masses. We carried out these calculations to avoid interpolations and more particularly, extrapolations. The importance of this procedure will be discussed later.

\section{Method}

DLEBS seem, at first glance, the ideal objects to compare with the predictions of theoretical stellar models. The traditional test requires theoretical isochrones to match the observed masses, radii and effective temperatures for a single chemical composition and age. Though these objects are clearly the most reliable sources of data on stellar masses and radii, other astrophysical parameters such as effective temperatures and chemical composition are not determined with the same precision. As an example, we can quote the empirical determination of the chemical composition. Even if we accept that the observed values of $Z$ are free from systematic errors, we should recall that stellar evolution also depends on the hydrogen (or helium) content. Therefore, if the observed values of $Z$ and $X$ are not determined simultaneously, a semi-empirical enrichment law must be used, which obviously yields additional uncertainties. This of course increases the number of free parameters involved in the investigation of DLEBS.

Another serious limitation relates to the determination of effective temperatures and their error bars. The absolute values of these astrophysical parameters are highly dependent on calibrations, on distances (the adopted extinction law), and on model atmospheres (uncertainties in the opacities, in the plane parallel approximation, and in the chemical composition). To avoid such inconveniences we decided to use the ratio of effective temperatures, TR $\equiv T_{\text {eff } 2} / T_{\text {eff1 }}$, instead of the effective temperatures themselves. This is a much better constrained parameter since it is directly derived from the light curve analysis and is independent of calibrations, distances, and atmosphere models. A similar procedure was used by Claret \& Willems (2002) and Claret (2003) for some problematic systems to avoid the uncertainties 
resulting from calibration/model dependencies. We used the following constraints to check whether the stellar models are able to reproduce the stellar parameters and, simultaneously, to estimate $\alpha_{\text {ov }}$ : a) the radii and masses of both components should be matched by the same isochrone, b) the effective temperature ratio should also be matched by the same isochrone as well as the apsidal-motion constant (when available). Summarizing, we analyse the systems using isochrones in the mass-radius and mass-TR diagrams simultaneously, or equivalently, for models computed for the precise observed masses, age-TR and age$\log g_{1,2}$ (in such diagrams an isochrone is represented by a vertical line). The systems that will be investigated are listed in Table 1, together their absolute dimensions and references. The error bars associated with TR were taken from the original papers. However, the error bars of the surface fluxes ratio are not available for two systems, namely SZ cen and YZ Cas. We estimated for these two systems a conservative mean error of around $1 \%$.

\section{Analysis of the individual systems}

The main difference between standard models and models taking into account core overshooting is the enlargement of the convective core. As a consequence more fuel is provided to be burnt, extending the life of the stars. The differences between standard models and those with core overshooting are small for systems lying near the ZAMS. However, when both components of a binary system begin to evolve, the differences become more and more noticeable. Therefore, to be a good test for the core overshooting amount, a system must show at least moderately evolved components.

An inspection of the data contained in Table 1 (see also Fig. 1) shows that some systems are not very evolved (V3903 Sgr and U Oph). The reason for including the first case in this study is that it is the most massive system with good determination of their absolute dimensions. We are interested in investigating the role of the mass on the amount of convective overshooting, and they are the only systems available for this mass range (20$\left.30 M_{\odot}\right)$. The second system is included for a similar reason: there are very few systems with masses in the region of $5 M_{\odot}$ with good determination of their absolute dimensions. Moreover, this system was recently reanalysed together with other three systems with similar masses (Vaz et al. 2007). The implications of this new analysis on the present paper will be discussed in Sect. 4.9.

Eight systems are common to those selected by Ribas et al. (2000): HV 2274, V380 Cyg, V906 Sco, WX Cep, V1031 Ori, SZ Cen, AI Hya, and TZ For. In addition to these eight systems and those described in the previous paragraph, we also investigate V453 Cyg, $\chi^{2}$ Hya and YZ Cas. The last two systems are well evolved and present an additional advantage: their mass ratios are not near 1.0, which allow us to test more strongly the differential evolution of models with variable core overshooting. In the specific case of YZ Cas, as the less massive component has a mass of $1.35 M_{\odot}$ it is possible to obtain two different values of $\alpha_{\text {ov }}$ for one system. On the other hand, V453 Cyg presents masses comparable with those of V380 Cyg but its absolute dimensions are around four times more accurate. Moreover, such a system is located in the open cluster NGC 6871 which can be used as an extra constraint. These three systems fulfil the requirements mentioned in the first paragraph. Unfortunately, Ribas et al. (2000) did not analyse them so a comparison with our results is not possible.

\subsection{TZ For}

This system presents very similar components but given their evolutionary status, the differential evolution can be detected. Previous studies have indicated that the more massive component is in a core helium burning phase (Claret \& Giménez 1995; Pols et al. 1997) whilst the secondary is near the TAMS (models with $\alpha_{\mathrm{ov}}=0.25$ ). As discussed by Pols et al. this solution has the attraction that it means the primary is in a long-lived evolutionary phase, whilst simultaneously being able to explain the circular orbit of TZ For (Claret \& Giménez 1995). We computed specific models for this system taking into account a variable amount of core overshooting $\alpha_{\mathrm{ov}}=0.0,0.2,0.4$, and 0.6 . The adopted chemical composition was $(X, Z)=(0.71,0.018)$. The results can be seen in Fig. 2. In order to evaluate the influence of the error bars in the derivation of the ages, we drew in the lower panels the track of the primary taking into account the error in its mass. For the sake of clarity we have plotted only the track for the primary, affected by the error bar. The conclusions are not clear. Standard models seems to fit the observations at the same age, provided that the errors in the mass measurements are considered, as well as the solutions adopting core overshooting.

The more clear solution is that of $\alpha_{\mathrm{ov}}=0.6$, which matches the TR and the observed $\log g$ values for the same age (even without considering the measurement error in the masses) and place the less massive component on the main sequence. This value seems to be too large for the mass range of TZ For. Indeed, neither Claret \& Giménez (1995), Pols et al. (1997), or Lastennet $\&$ Valls-Gabaud (2002) found good fits to the properties of this system. Only marginal solutions could be found. As TZ For is a very evolved system, perhaps this unclear scenario could be atributted to deficiencies in stellar modelling, mainly in the later phases of evolution.

\subsection{Al Hya}

AI Hya is another system with similar components, but with masses around $2 M_{\odot}$. The situation of this binary is little more clear than for TZ For. For $Z=0.02$ and $X=0.71$ we can find a good fit only by assuming $\alpha_{\mathrm{ov}}=0.2$ and 0.25 (Fig. 3). For models without overshooting or with $\alpha_{\text {ov }}$ greater than 0.4 the fits are not so good, and are marginally within 1-2 $\sigma$.

\section{3. $Y Z$ Cas}

YZ Cas is a very interesting system because it has a mass ratio of approximately 0.6. As mentioned before, this allows us to test its differential evolution in more detail. Figure 4 shows the models for this system. The best solutions are those with no core overshooting and with $\alpha_{\mathrm{ov}}=0.2$. These solutions put the two components on the main sequence. Although the solutions for greater overshooting are within the observational errors the previous prescriptions fit the observational data better. One can also explore the mass of the secondary star, $1.35 M_{\odot}$. It is expected that a smaller amount of overshooting is sufficient to match it and in this case one would have a system with different values of $\alpha_{\mathrm{ov}}$. We have computed some models with varying core overshooting for the secondary (keeping the chemical composition constant) and have found that $\alpha_{\mathrm{ov}}=0.1$ provides a simultanenous match for its TR and $\log g$ values. Unfortunately, the secondary is not very evolved and these models are almost indistinguinshable from those adopting $\alpha_{\mathrm{ov}}=0.0$ and 0.2 . 
Table 1. Astrophysical parameters for the sample of seleted DLEBS.

\begin{tabular}{|c|c|c|c|c|c|c|c|c|c|c|c|}
\hline Name & $\overline{m_{1}}$ & $\overline{m_{2}}$ & 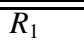 & 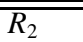 & $\overline{\log g_{1}}$ & $\overline{\log g_{2}}$ & $\overline{\log T_{\text {eff } 1}}$ & $\overline{\overline{\log T_{\text {eff2 }}}}$ & $\overline{\overline{\alpha_{\mathrm{ov}}}}$ & $\overline{\overline{T R R}}$ & Ref. \\
\hline \multirow[t]{2}{*}{ V3903 Sgr } & 27.27 & 19.01 & 8.088 & 6.125 & 4.058 & 4.143 & 4.580 & 4.533 & $0.3 \pm 0.2$ & $0.899 \pm 0.006$ & 1 \\
\hline & 0.55 & 0.44 & 0.086 & 0.060 & 0.016 & 0.013 & 0.022 & 0.022 & $0.3 \pm 0.2$ & & \\
\hline \multirow[t]{2}{*}{ HV 2274} & 12.2 & 11.4 & 9.86 & 9.03 & 3.536 & 3.585 & 4.362 & 4.364 & $0.3_{-0 .}^{+0.3}$ & $1.005 \pm 0.005$ & 2 \\
\hline & 0.7 & 0.7 & 0.24 & 0.24 & 0.027 & 0.029 & 0.003 & 0.003 & $0.3^{+0.3}$ & & \\
\hline \multirow[t]{2}{*}{ V453 Cyg } & 14.36 & 11.11 & 8.551 & 5.489 & 3.731 & 4.005 & 4.425 & 4.407 & $0.2 \pm 0.1$ & $0.959 \pm 0.014$ & 3 \\
\hline & 0.20 & 0.13 & 0.055 & 0.063 & 0.012 & 0.015 & 0.008 & 0.014 & $0.2 \pm 0.1$ & & \\
\hline \multirow[t]{2}{*}{ V380 Cyg } & 11.1 & 6.95 & 14.7 & 3.74 & 3.148 & 4.133 & 4.329 & 4.312 & $0.4_{-0.3}^{+0.2}$ & $0.96 \pm 0.02$ & 4 \\
\hline & 0.5 & 0.25 & 0.2 & 0.07 & 0.023 & 0.023 & 0.007 & 0.011 & $0.2 \pm 0.1$ & & \\
\hline \multirow[t]{2}{*}{ U Oph } & 5.273 & 4.738 & 3.483 & 3.109 & 4.0682 & 4.128 & 4.2159 & 4.1928 & $0.2_{-01}^{+0.2}$ & $0.9481 \pm 0.0003$ & 5 \\
\hline & 0.091 & 0.072 & 0.020 & 0.034 & 0.0098 & 0.012 & 0.0066 & 0.0070 & $0.2_{-0.2}^{+8: 2}$ & & \\
\hline \multirow[t]{2}{*}{$\chi^{2}$ Нya } & 3.613 & 2.638 & 4.384 & 2.165 & 3.712 & 4.188 & 4.066 & 4.041 & $0.2_{-02}^{+0.1}$ & $0.945 \pm 0.004$ & 6 \\
\hline & 0.079 & 0.050 & 0.039 & 0.043 & 0.015 & 0.019 & 0.010 & 0.010 & $0.2_{-0.2}^{+0.11}$ & & \\
\hline \multirow[t]{2}{*}{ V906 Sco } & 3.253 & 3.378 & 3.515 & 4.521 & 3.858 & 3.656 & 4.029 & 4.017 & $0.2_{-02}^{+0 . \bar{I}}$ & $0.972 \pm 0.002$ & 7 \\
\hline & 0.069 & 0.071 & 0.039 & 0.035 & 0.013 & 0.012 & 0.020 & 0.020 & $0.2_{-0.2}^{+0.11}$ & & \\
\hline \multirow[t]{2}{*}{ WX Cep } & 2.539 & 2.329 & 3.994 & 2.711 & 3.640 & 3.939 & 3.911 & 3.949 & $0.1 \pm 0.1$ & $1.09 \pm 0.02$ & 8 \\
\hline & 0.050 & 0.045 & 0.030 & 0.023 & 0.011 & 0.011 & 0.012 & 0.012 & $0.1 \pm 0.1$ & & \\
\hline \multirow[t]{2}{*}{ V1031 Ori } & 2.473 & 2.286 & 4.321 & 2.977 & 3.560 & 3.850 & 3.895 & 3.924 & $0.15 \pm 0.05$ & $1.069 \pm 0.002$ & 9 \\
\hline & 0.018 & 0.016 & 0.034 & 0.064 & 0.008 & 0.019 & 0.028 & 0.026 & $0.15 \pm 0.05$ & & \\
\hline \multirow[t]{2}{*}{ SZ Cen } & 2.317 & 2.277 & 4.554 & 3.624 & 3.486 & 3.677 & 3.857 & 3.892 & $0.1 \pm 0.1$ & $1.08 \pm 0.01$ & 10 \\
\hline & 0.026 & 0.021 & 0.032 & 0.026 & 0.008 & 0.007 & 0.017 & 0.017 & $0.1 \pm 0.1$ & & \\
\hline \multirow[t]{2}{*}{ YZ Cas } & 2.314 & 1.350 & 2.534 & 1.348 & 3.995 & 4.309 & 3.964 & 3.826 & $0.2_{-0.15}^{+0.1}$ & $0.73 \pm 0.01$ & 11 \\
\hline & 0.021 & 0.010 & 0.026 & 0.015 & 0.011 & 0.010 & 0.014 & 0.016 & $0.05 \pm 0.05$ & & \\
\hline \multirow[t]{2}{*}{ AI Hya } & 2.145 & 1.978 & 3.194 & 2.766 & 3.584 & 3.850 & 3.826 & 3.851 & $0.2 \pm 0.05$ & $1.06 \pm 0.06$ & 12 \\
\hline & 0.038 & 0.036 & 0.031 & 0.017 & 0.011 & 0.010 & 0.004 & 0.004 & $0.2 \pm 0.05$ & & \\
\hline \multirow[t]{2}{*}{ TZ For } & 2.049 & 1.949 & 8.318 & 3.962 & 2.909 & 3.532 & 3.699 & 3.803 & $0.4_{-03}^{+0.2}$ & $1.27 \pm 0.01$ & 13 \\
\hline & 0.055 & 0.027 & 0.121 & 0.088 & 0.018 & 0.020 & 0.009 & 0.007 & $0.4_{-0.3}^{+0.2}$ & & \\
\hline
\end{tabular}

References: 1 Vaz et al. (1997); 2 Ribas et al. (2000); 3 Southworth et al. (2004); 4 Guinan et al. (2000); 5 Vaz et al. (2007); 6 Clausen \& Nordström (1978); 7 Alencar et al. (1997); 8 Popper (1987); 9 Andersen A. et al. (1990); 10 Andersen (1975); 11 Lacy (1981); 12 Popper (1988); 13 Andersen et al. (1991).

\subsection{SZ Cen}

We found solutions for $(X, Z)=(0.70,0.02)$, which are presented in Fig. 5. As our selected chemical composition for SZ Cen coincides with one of our previously computed grids, we adopt a different format for the corresponding figure. The conclusions are similar to the previous systems with masses around $2 M_{\odot}$ and we adopt as the best solution the range 0.0-0.2 for $\alpha_{\mathrm{ov}}$.

\subsection{V1031 Ori}

The standard models do not match well the observed TR and the surface gravities of V1031 Ori, as can clearly be seen in Fig. 6. The best fitting is achieved with $\alpha_{\mathrm{ov}}=0.2$, though acceptable solutions are also possible for greater core overshooting. However, when we increase $\alpha_{\mathrm{ov}}$, the agreement gets worse. Therefore, we adopt 0.15 as the best value for $\alpha_{\mathrm{ov}}$. The chemical composition is perfectly within the expected values of the primordial helium abundance and the enrichment law mentioned above.

\subsection{WX Cep}

As in the case of SZ Cen, the selected chemical composition coincides with one of our previously computed grids. The results are plotted in Fig. 7 (where only the models with $\alpha_{\text {ov }}=0.2$ are shown). The best solutions are for $\alpha_{\mathrm{ov}}=0.0$ and 0.2 .

\subsection{V906 Sco}

This system was analysed by Alencar et al. (1997) who determined that this triple star is a member of the open cluster M 7 and has individual stellar masses around 3.4 $M_{\odot}$. The precison of its absolute dimensions is better than $2 \%$. When comparing its absolute dimensions to theoretical predictions, we found good agreement with the analysis performed by the above authors concerning the age and amount of convective core overshooting, though they used older stellar models. We found that the standard models (Fig. 8) as well as models with moderate core overshooting provide acceptable matches. For $\alpha_{\mathrm{ov}}$ larger than 0.4 , the models fails to predict correctly the effective temperature ratio. As an extra constraint, we have fitted isochrones to the $u v b y$ data for M 7, finding a good interagreement with the age derived by the above mentioned authors (250 Myr) and also with the inferred age for V906 Sco using moderate core overshooting.

\section{8. $\chi^{2}$ Hya}

Like YZ Cas, $\chi^{2}$ Hya also presents a relatively low mass ratio (0.73), which allows a more critical comparison of its observational data with theoretical predictions. The primary star is almost $50 \%$ more massive than those in the previous systems. The observational data of both components are compatible with no core overshooting and with $\alpha_{\mathrm{ov}}=0.2,0.4$, and 0.6 (Fig. 9). However, the fit for $\alpha_{\mathrm{ov}}=0.6$ is not as good as those for $\alpha_{\mathrm{ov}}=0.0,0.2$, and 0.4. A more careful inspection of the figure indicates that we can fix as an upper limit $\alpha_{\mathrm{ov}} \approx 0.30$. This system was not investigated by Ribas et al. (2000) and a direct comparison is not possible.

\subsection{U Oph}

This mid B-type DLEBS was recently reanalysed, using new radial velocities and uvby light curves, by Vaz et al. (2007). The resulting masses and radii are very precise: less than $2 \%$ for mass 
and around $0.5 \%$ for the radius. This means that the mean accuracy is 3 times larger than, for example, V380 Cyg. This level of accuracy permits us to test evolutionary models with more confidence than for other systems. Moreover, as U Oph is in a mass range with very scarce members $\left(5 M_{\odot}\right)$ it is important to include it to avoid a bias in mass when we attempt to establish a relationship between mass and $\alpha_{\mathrm{ov}}$. In addition to these points, in the quoted paper the authors also analysed the case of three DLEBS with similar masses (DI Her, MU Cas and V760 Sco), and this is why we introduced U Oph in this study. The best fitting is achieved with $\alpha_{\mathrm{ov}}=0.2$. For higher amounts of core overshooting, the models fails to predict the effective temperatures ratio. We refer interested readers to the original paper by Vaz et al. (2007).

\subsection{V380 Cyg}

This system is fundamental to our purpose since its primary is a relatively evolved star and its mass ratio is 0.63 . For this system it is possible to investigate more aspects of differential evolution. Its absolute dimensions are known with a good level of accuracy (but not at the same level of other systems of our sample) and the system also shows apsidal motion. In Fig. 2 by Ribas et al. (2000), it is clear that V380 Cyg is key to defining the general trend of the $\log M$ versus $\alpha_{\text {ov }}$ diagram since the other system used by Ribas et al. (2000) in this mass range, HV 2274, has similar components and it is not useful for a critical test of the strength of core overshooting.

Ribas et al. claim that, on the basis of the work by Guinan et al. (2000), a large amount of core overshooting is needed to match the observational properties of V380 Cyg. They determined $\alpha_{\mathrm{ov}}=0.6 \pm 0.1$ as the best solution. This value, however, seems not to be in agreement with independent astrophysical sources. The analyses of several DLEBS carried out by Pols et al. (1997), Claret \& Willems (2002) and Lastennet \& VallsGabaud (2002) do not support such a high value. Isochrone fits to the colour-magnitude diagrams of clusters, mainly the younger ones, also do not support such a high value of $\alpha_{\mathrm{ov}}$. Several clusters were observed recently with the $\Delta a$ photometric system, which is designed to detect chemically peculiar stars, and it was found that $\alpha_{\mathrm{ov}}=0.2$ is sufficient to match the observations (Paunzen et al. 2006, and references given therein). Moreover, as discussed by Claret (2003) the error bars in the effective temperatures of V380 Cyg, which were key to the determination of $\alpha_{\text {ov }}=0.6$, seem to be optimistic.

From these considerations, Claret (2003) was able to show that even standard models are able to match the properties of V380 Cyg (provided that TR is used as a constraint, instead of the effective temperatures themselves), finding $\alpha_{\mathrm{ov}}=0.6_{-0.4}^{+0.1}$. As commented in the mentioned paper, we have some reservations concerning this value. In fact, we consider the range of possible values of $\alpha_{\mathrm{ov}}$ to be more important than the central value itself (0.6).

The apsidal motion of V380 Cyg can be used to investigate the best value of $\alpha_{\mathrm{ov}}$. However, one should recall that apsidal motion is a test of stellar evolution, not a strong constraint. However, it is a useful guide. The lower panels of Fig. 10 show how $\log \overline{k_{2}}$ depends on time for $\alpha_{\mathrm{ov}}=0,0.2,0.4$, and 0.6. The relativistic correction (which is around $12 \%$ of the total apsidal motion rate) was taken into account. Let us consider the influence of apsidal motion on the determination of the best value of the core overshooting parameter. Except for the standard models, all models match well the observed apsidal motion rate of
V380 Cyg (shown as a horizontal dashed lines in the lower panels of Fig. 10). We can conclude that the error bars are not symmetrical and that $\alpha_{\mathrm{ov}}=0.4_{-0.3}^{+0.2}$, which is different from that derived previously due mainly to the new input phsyics of the models. This indicates the level of uncertainty we are dealing with.

\subsection{V453 Cyg}

V453 Cyg is another fundamental system for the definition of the $\log M$ versus $\alpha_{\text {ov }}$ relationship. Southworth et al. (2004) used new high-resolution spectroscopy and published UBV light curves to derive very accurate values for the masses and radii of V453 Cyg (the mean error is $1.4 \%$ ). This is a very interesting DLEBS for several reasons: the component masses are very near of those of V380 Cyg, it displays apsidal motion, and it is located in the open cluster NGC 6871. These authors compared the absolute dimensions of V453 Cyg with four different set of stellar models: Granada, Padova, Geneva, and Cambridge. They found good inter-agreement among the four sets of evolutionary models, with a mean age of $10.0 \pm 0.2 \mathrm{Myr}$ and $(X, Y)=(0.70,0.02)$. They also found that the models with moderate core overshooting $\left(\alpha_{\mathrm{ov}}=0.2\right)$ fit the observational data slightly better than standard models. No extra mixing beyond $\alpha_{\mathrm{ov}}=0.2$ was needed to match the masses, radii and effective temperatures. In addition, the theoretical apsidal motion rate predicted by the Granada models was found to be in very good agreement with the observed rate.

Our models for V453 Cyg are consistent with the general conclusions by Southworth et al. (2004). In Fig. 11 we show the time evolution of our models with varying amounts of core overshooting (from 0.0 up to 0.6). The derived ages are between 10 Myr for standard models and 13 Myr for models with $\alpha_{\mathrm{ov}}=0.6$, the fit being worse for larger amounts of core overshooting. Again, the standard models or models with moderate core overshooting cannot be ruled out since they provide acceptable solutions. The relativistic contribution to the apsidal motion rate (around $6 \%$ of the total) was accounted for. The theoretical $\log \overline{k_{2}}$ was computed by assuming that the period and the eccentricity were constant (at the observed values). This is not necessarily correct due to secular changes in the orbital elements, but near the region of interest it is essentially a good approximation. The influence of both stars are considered to derive $\log \overline{k_{2}}$. The comparison with the observed apsidal motion rate leads to the same conclusions as the comparison with the absolute dimensions: it is not possible to discard the standard models or those with moderate core overshooting since they also give an acceptable match to the observations. A complementary test is the comparison of the colour-magnitude diagram of NGC 6871 with theoretical predictions. An age of $10 \mathrm{Myr}$ is found for a moderate core overshooting, in good agreement with the ages derived from the comparison of thereotical models and the absolute dimensions of V453 Cyg.

\subsection{HV 2274}

HV 2274 is not a good test for core overshooting due two main causes: 1) the masses of the componets are nearly equal and 2) the associated errors in masses and radii are too large. However, due to its strategic position in the HR diagram, we decided to include it. As expected, HV 2274 can be matched by more than one chemical composition/core overshooting parameter. For a fixed chemical composition, $(X, Z)=(0.739,0.007)$, we found that the range of the best $\alpha_{\mathrm{ov}}$ is between 0.2 and 0.6 . 


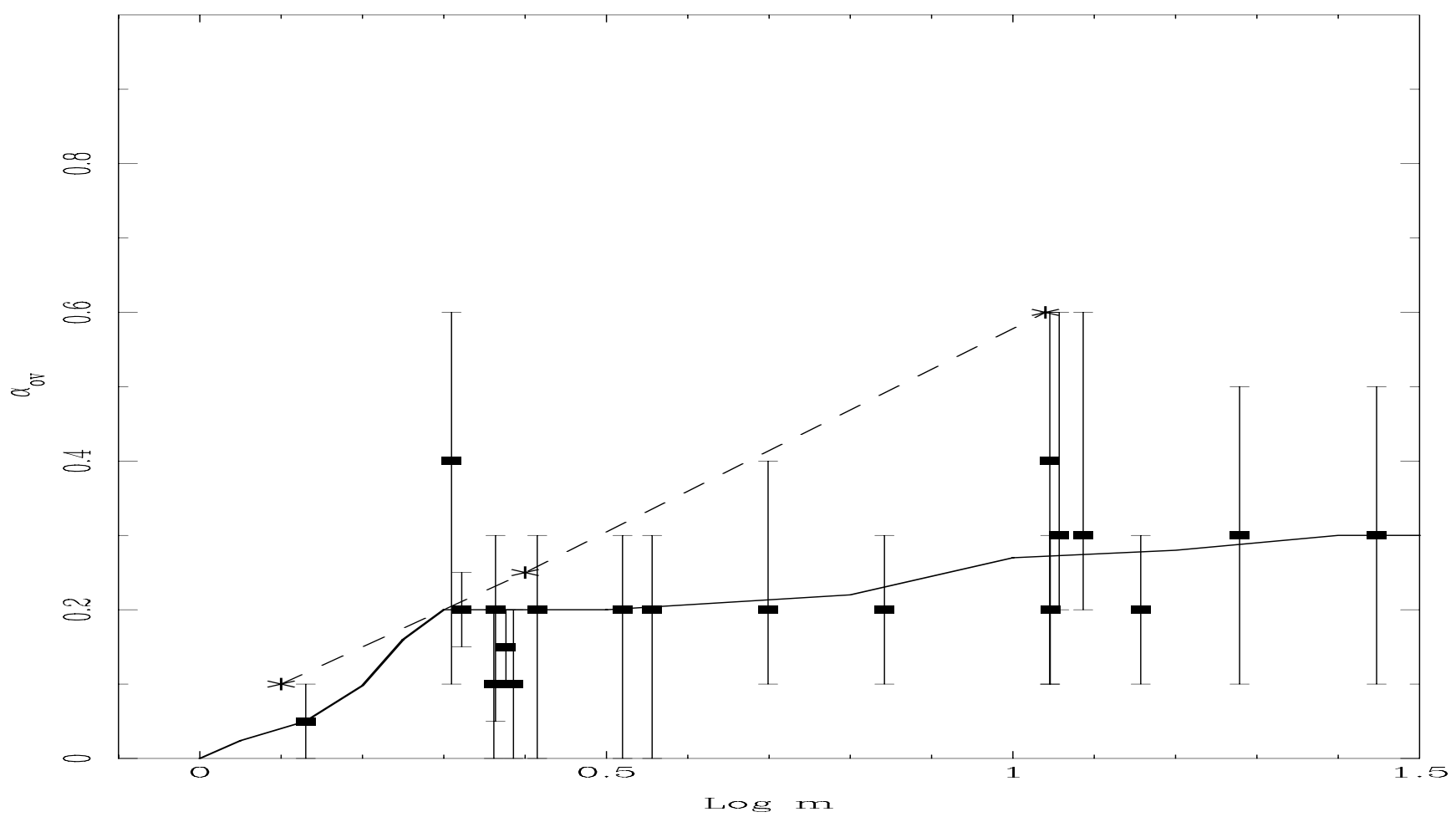

Fig. 13. The dependence of the amount of core overshooting with the logarithm of mass (in solar units). The continuous thick line (full rectangles and error bars) represents the results obtained in the present paper while dashed line (asterisks) denotes the relationship established by Ribas et al. (2000).

\subsection{V3903 Sgr}

This is the most massive system of our sample. It was selected due to this and also due to its low mass ratio, which is around 0.70 . In principle, the system could be an excellent test to out present purpose. However as explained before, it is a very young system and the resulting conclusions are limited. Despite this, we decided to include it to better cover the range of stellar masses in our analysis. Vaz et al. (1997) found good agreement with the predictions of old models computed with moderate overshooting ( $\alpha_{\mathrm{ov}}=0.2$ ), even including the absolute values of the effective temperatures. As these authors derived more reliable error bars in effective temperatures we decided to use them to establish the best fit. We found acceptable matches from $\alpha_{\text {ov }}=0.0$ up to 0.6 for $(X, Z)=(0.70,0.02)$. Only tracks with $\alpha_{\mathrm{ov}}=0.4$ are shown in Fig. 12 . Note that the fitting using $\alpha_{\mathrm{ov}}=0.4$ is only marginally acceptable concerning TR.

\section{Comparision with previous works: the unclear scenario of the mass dependence of core overshooting}

The results of our comparisons between the observational data for the DLEBS displayed in Table 1, and the theoretical stellar models with varying core overshooting, are far from conclusive. There seems to be a real tendency for $\alpha_{\text {ov }}$ to increase with mass (Fig. 13), but the associated uncertainties are still large (although more realistic than previously). Moreover, it was not possible to separate the effects of chemical composition in the analysis.

Unfortunately, as far as we know, there is only one previous paper, Ribas et al. (2000), which is devoted to an investigation of the dependence of convective core overshooting on mass using eclipsing binaries. Some signficant differences can be found between their paper and the work presented here. The first concerns the use of the absolute values of the effective temperatures. As stressed by Claret (2003), such parameters are not free from calibrations and are subject to several uncertainties concerning stellar atmosphere models, the adopted extinction law and for some specific cases (e.g. V380 Cyg), the low luminosity of the secondary components. This is why we decided to adopt the effective temperature ratio, instead of the absolute values.

Another important difference refers to the adopted chemical compositions. In this paper we have tried to keep the helium content to within the limits given by an acceptable enrichment law. For some DLEBs Ribas et al. adopted values of $Y$ which were too low, some of them being smaller than the primordial helium abundance, for example the cases of SZ Cen, V1031 Ori and WX Cep. The low values of $Y$ found by these authors maybe attributed to their fitting algorithm and/or the direct use of the absolute effective temperatures, which in some cases can yield low values of $Y$.

Differences can also be found concerning the number of points used to establish the mass versus $\alpha_{\mathrm{ov}}$ relationship (Fig. 13). We have plotted all available information from our comparison, including the best value of $\alpha_{\mathrm{ov}}$ for the secondaries when the mass ratio is very different to 1 . Two systems are not sufficiently evolved to be a good test of the amount of core overshooting but, as their masses are in a strategic range, they were included in our analysis for completeness. Three suitable evolved systems, not used by Ribas et al., were also studied: V453 Cyg, $\chi^{2}$ Hya and YZ Cas. As a starting point for our analysis we considered the possibility that standard models could also match the observations, and indeed we found that for some systems $\alpha_{\mathrm{ov}}=0.0$ provides a good solution.

A recent investigation on the influence of the core overshooting on the isochrones was carried out by VandenBerg et al. (2006). In order to compute the stellar models they infer, using mainly CMD of clusters, a relationship between mass and core 
overshooting amount which seems to be different from that derived in the present paper. However, the parametrization of core overshooting amount used in the models by VandenBerg et al. (2006) is different from that used here and the free parameter $F_{\text {ov }}$ is not directly comparable with our $\alpha_{\text {ov }}$. On the other hand, in order to check whether an extra mixing is required to fit the CMD of some key clusters, we computed isochrones for M 67, NGC 752 and NGC 3680 and found good agreement with those by Sarajedini et al. (1999) and Kozhurina-Platais et al. (1997). These results seem to indicate that there is no need for an extra mixing beyond $\alpha_{\mathrm{ov}}=0.2$, in good agreement with the results described in Paunzen et al. 2006 (and references therein).

The comparison of several DLEBS with evolutionary models also indicates that a moderate amount of core overshooting, $\alpha_{\text {ov }}$ typically of the order of 0.2 , is sufficient to match the obervational data (Pols et al. 1997; Lastennet \& Valls-Gabaud 2002; Claret \& Willems 2002). As a consequence, the relationship we found between mass and $\alpha_{\mathrm{ov}}$ shows a less pronounced slope (continuous thick line in Fig. 13) than previously established by Ribas et al., who used only three points to define their relationship (asterisks). The mass dependence which we inferred will probably require modification when new input physics and/or more accurate absolute dimensions become available. Of particular interest, from the theoretical point of view, is the new generation of opacity tables and the effects of induced mixing by rotation.

Acknowledgements. I thank J. V. Clausen for his comments and suggestions that improved the paper. I am also in debt with J. Southworth for his comments. The Spanish MEC (AYA2006-06375) is gratefully acknowledged for its support during the development of this work. Use was made of the WEBDA database, operated at the University of Vienna, Austria.

\section{References}

Alencar, S. P., Vaz, L. P. R., \& Helt, B. E. 1997, 326, 709

Andersen, A. 1975, A\&A, 45, 203

Andersen, A., \& Clausen, J. V. 1989, A\&A, 213, 183

Andersen, A., Clausen, J. V., \& Nordström, B. 1990, A\&A, 228, 365

Andersen, A., Clausen, J. V., Nordström, B., Tomkin, J., \& Mayor, M. 1991, A\&A, 246, 99

Andersen, J., Nordström, B., \& Clausen, J. V. 1990, ApJ, 363, L33
Alexander, D. R., \& Ferguson, J. W. 1994, ApJ, 437, 879

Bressan, A. G., Fagotto, F., Bertelli, G., \& Chiosi, C. 1993, A\&AS, 100, 647

Clausen, J. V., \& Nordström, B. 1978, A\&A, 31, 307

Claret, A. 1995, A\&AS, 109, 441

Claret, A. 2004, A\&A, 424, 919

Claret, A. 2007, A\&A, 467, 1389

Claret, A., \& Giménez, A. 1991, A\&A, 244, 319

Claret, A., \& Giménez, A. 1993, A\&A, 277, 487

Claret, A., \& Giménez, A. 1995, A\&A, 296, 180

Claret, A., Giménez, A., \& Cunha, N. C. S. 1995, A\&A, 299, 724

Claret, A., \& Cunha, N. C. S. 1997, A\&A, 318, 187

Claret, A., \& Willems, B. 2002, A\&A, 388, 518

Formicola, A., et al. 2004, Phys. Lett. B, 591, 61

Guinan, E, Ribas, I., Fitzpatrick, E. L., et al. 2000, ApJ, 544, 409

Iglesias, C. A., \& Rogers, F. J. 1996, ApJ, 464, 943

Kozhurina-Platais, V., Demarque, P., Platais, I. Orosz, J. A., \& Barnes, S. 1997, AJ, 113, 1045

Kuhfuss, R. 1987, in Nuclear Astrophysics, ed. W. Hillebrandt, R. Kuhfuss, E. Mueller, \& J. W. Truran (Berlin: Springer-Verlag), 222

Lacy, C. H. 1981, ApJ, 251, 591

Lastennet, E., \& Valls-Gabaud, D. 2002, A\&A, 396, 551

Maeder, A. 1975, A\&A, 40, 303

Nieuwenhuijzen, H., \& de Jager, C. 1990, A\&A, 231, 134

Nugis, T., \& Lamers, H. J. G. L. M. 2000, A\&A, 360, 227

Paunzen, E., Netopil, M., Iliev, I. Kh., et al. 2006, A\&A, 454, 171

Pols, O. R., Tout, C. A., Eggleton P. P., \& Han, Z. 1995, MNRAS, 274, 964

Popper, D. M. 1987, AJ, 93, 672

Popper, D. M. 1988, AJ, 95, 190

Reimers, D. 1977, A\&A, 61, 217

Ribas, I., Jordi, C., \& Giménez, A. 2000, MNRAS, 318, 55

Rogers, F. J., \& Iglesias, C. A. 1992, ApJS, 79, 507

Runkle, R. C. 2003, Ph.D. Thesis, University of North Carolina, unpublished

Sarajedini, A., von Hippel, T., Kozhurina-Platais, V., \& Demarque, P. 1999, AJ, 118,2894

Saslaw, W. C., \& Schwarzschild, M. 1965, ApJ, 142, 1468

Schaller, G., Schaerer, D., Meynet, G., \& Maeder, A. 1992, A\&AS, 96, 269

Shaviv, G., \& Salpeter, E. E. 1973, ApJ, 184, 191

Southworth, J., Maxted, P. F. L., \& Smalley, B. 2004, MNRAS, 351, 1277

Stothers, R. B., \& Chao-wen Chin 1991, ApJ, 381, L67

Stothers, R. B., \& Chao-wen Chin 1992, ApJ, 390, 136

Straus, J. M., Blake, J. B., \& Schramm, D. N. 1976, ApJ, 204, 481

Umezu, M. 1995, MNRAS, 276, 1287

VandenBerg, D. A., Bergbusch, P. A., \& Dowler, P. D. 2006, ApJS, 162, 375

Vaz, L. P. R., Cunha, N. C. S., Vieira, E. F., \& Myrrha, M. L. M. 1997, A\&A, 327,1094

Vaz, L. P. R., Andersen, A., \& Claret A. 2007, A\&A, 469, 285

Xiong, D. R. 1985, A\&A, 150, 133

Xiong, D. R. 1986, A\&A, 167, 239 
A. Claret: Does convective core overshooting depend on stellar mass?, Online Material $p 1$

\section{Online Material}


A. Claret: Does convective core overshooting depend on stellar mass?, Online Material p 2

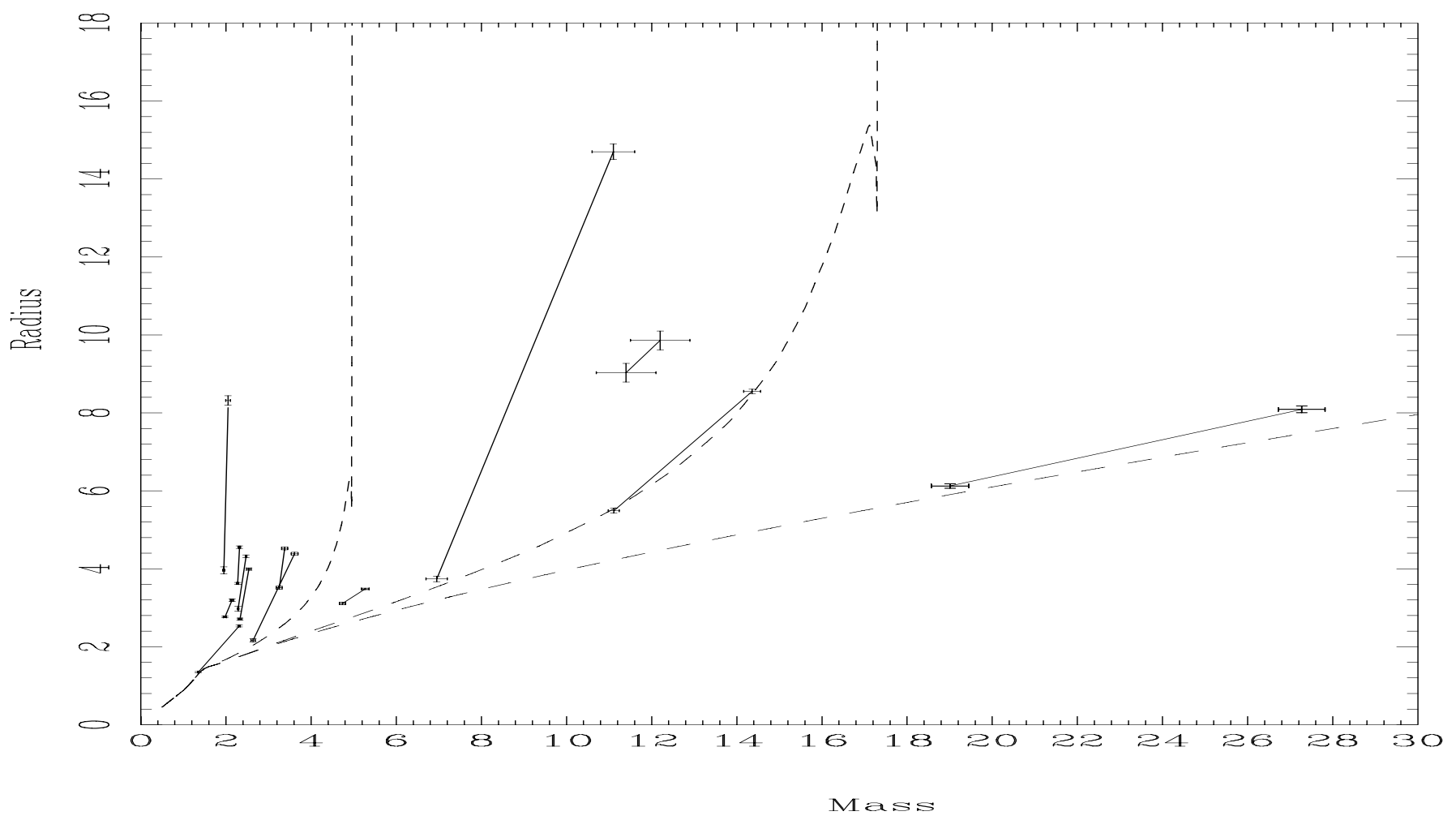

Fig. 1. Mass-radius diagram for the individual components (connected by line) of the binary systems in Table 1 . Theoretical isochones are also shown for reference as dashed lines. The ages are $1 \times 10^{6}, 1 \times 10^{7}$ and $1 \times 10^{8}$ years. $(X, Z)=(0.70,0.02)$ and $\alpha_{\mathrm{ov}}=0.2$.
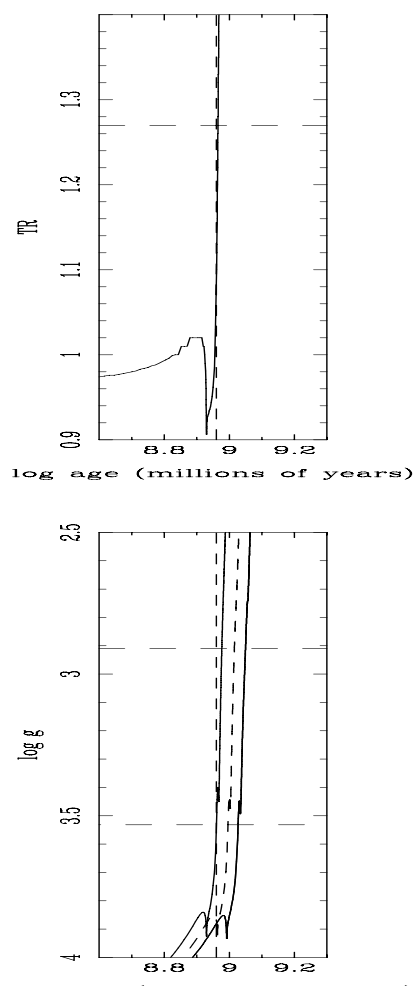
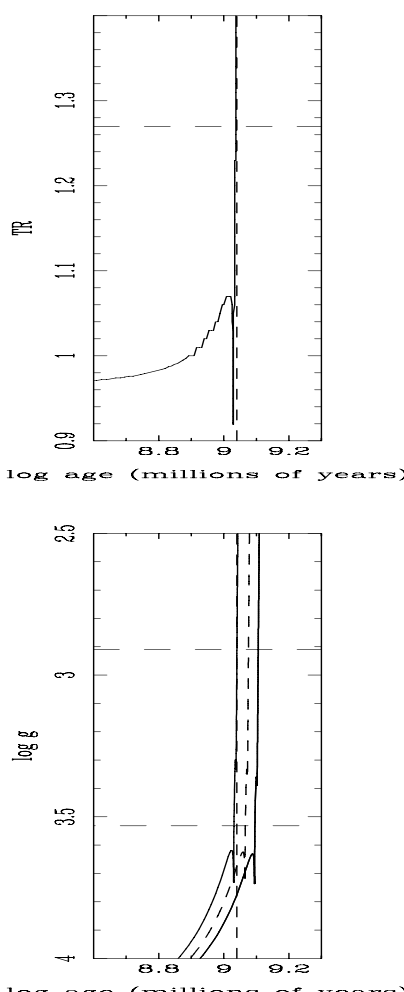
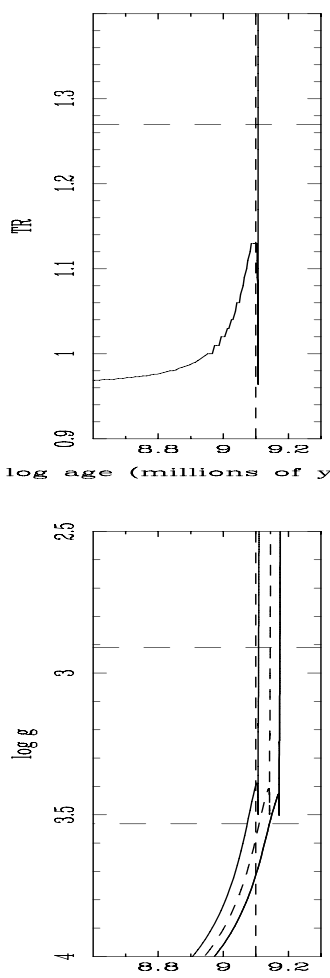
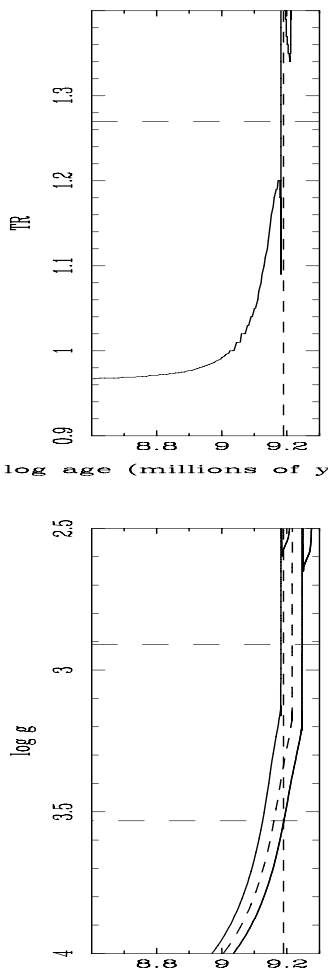

Fig. 2. The theoretical ratio of the effective temperatures TR (upper panels), the surface grativity for the two components (lower panels) of TZ For as a function of time. Solid lines denote the theoretical calculations while horizontal dashed lines indicate the observed values. The values of $\alpha_{\mathrm{ov}}$ are $0.0,0.2,0.4$, and 0.6 from the left to right. $(X, Z)=(0.71,0.018)$. 
A. Claret: Does convective core overshooting depend on stellar mass?, Online Material p 3
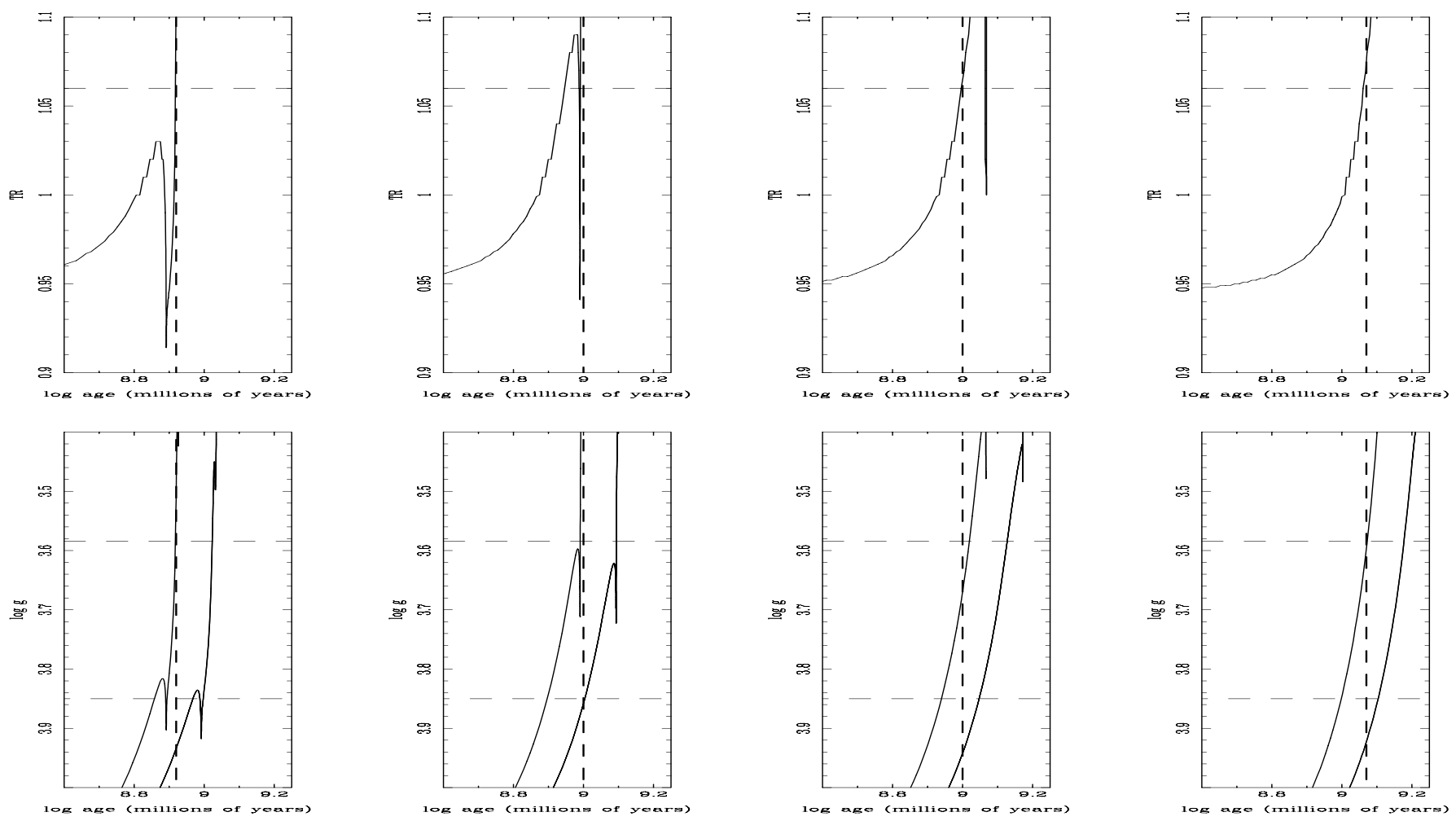

Fig. 3. The same as in Fig. 1 but for AI Hya. $(X, Z)=(0.71,0.02)$.
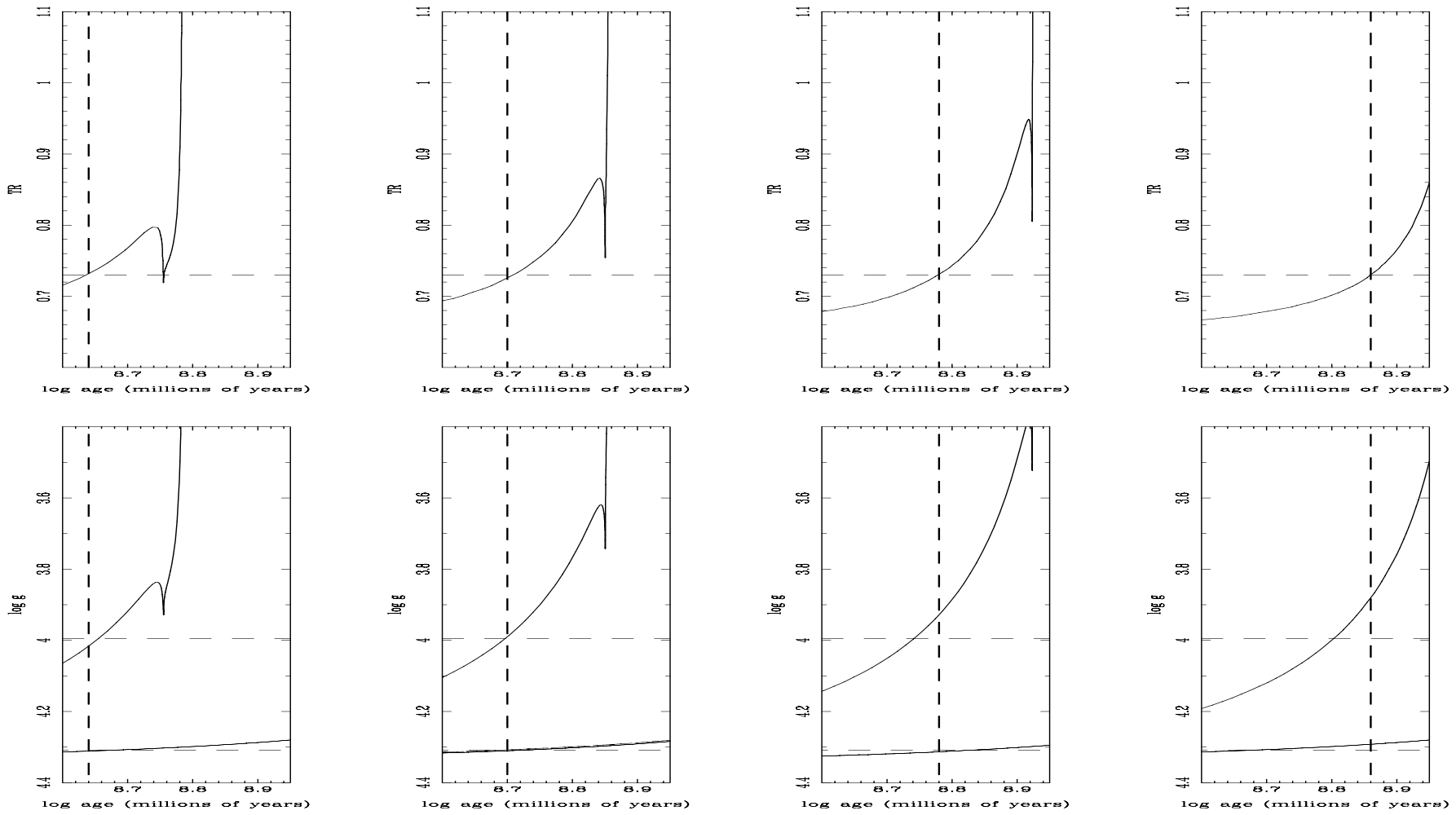

Fig. 4. The same as in Fig. 1 but for YZ Cas. $(X, Z)=(0.715,0.015)$. 
A. Claret: Does convective core overshooting depend on stellar mass?, Online Material p 4

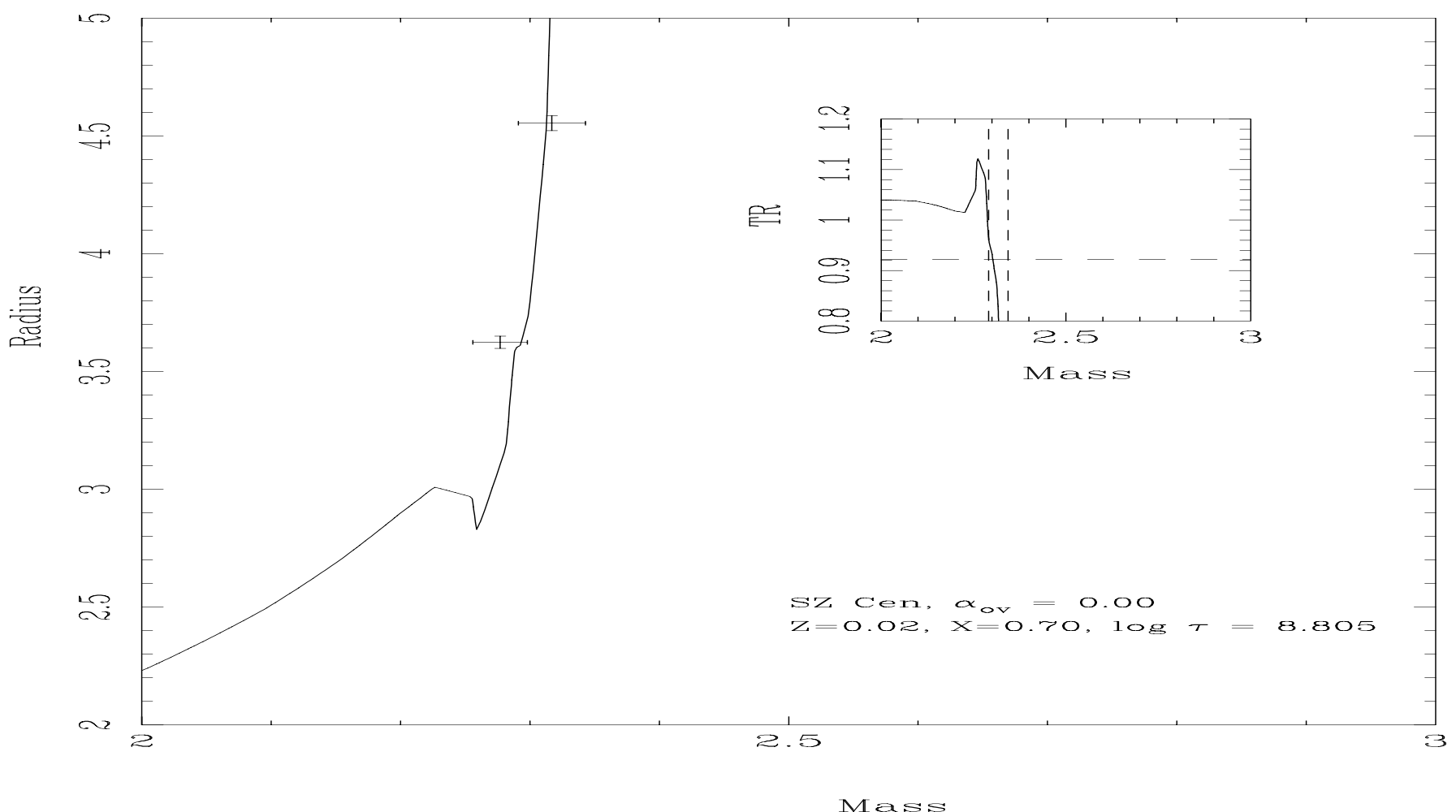

Fig. 5. Isochrones for SZ Cen. Main frame: mass-radius diagram. Small diagram: mass of the primary star versus TR (for this figure and for V3903 Sgr, V906 Sco, WX Cep, TR is redefined to be $\left.\equiv T_{\text {eff1 } 1} / T_{\text {eff2 }}\right)$. Only the case of no overshooting is shown. $(X, Z)=(0.70,0.02)$.
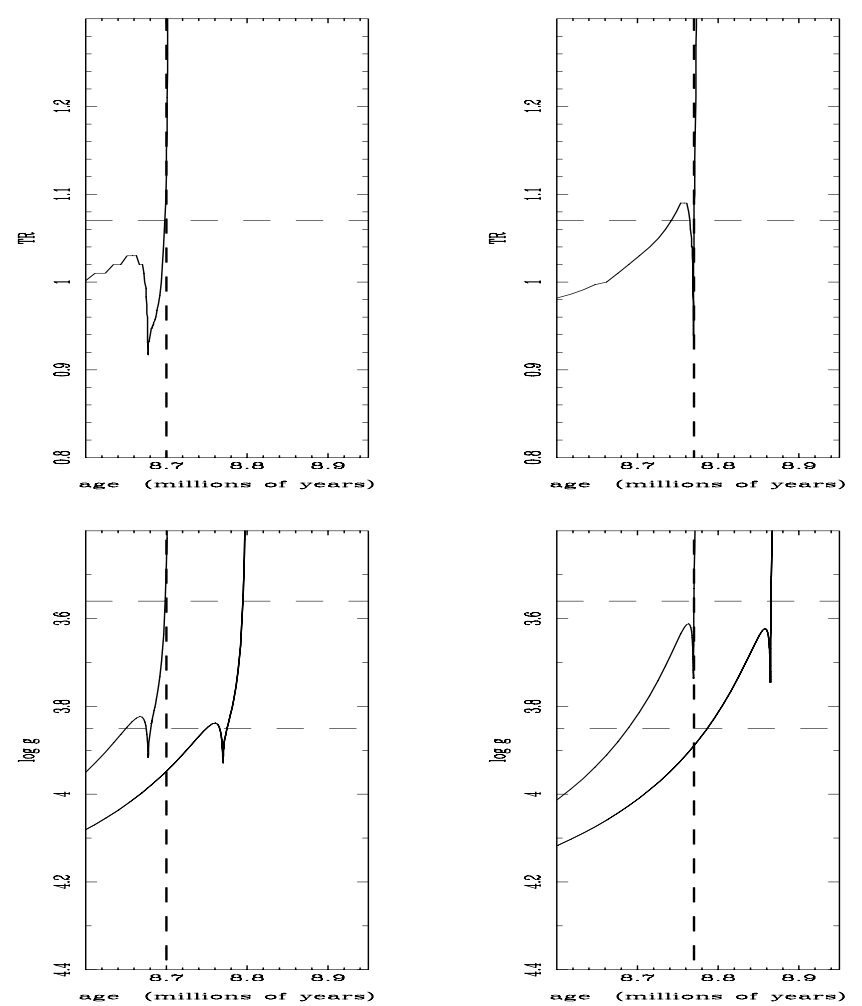
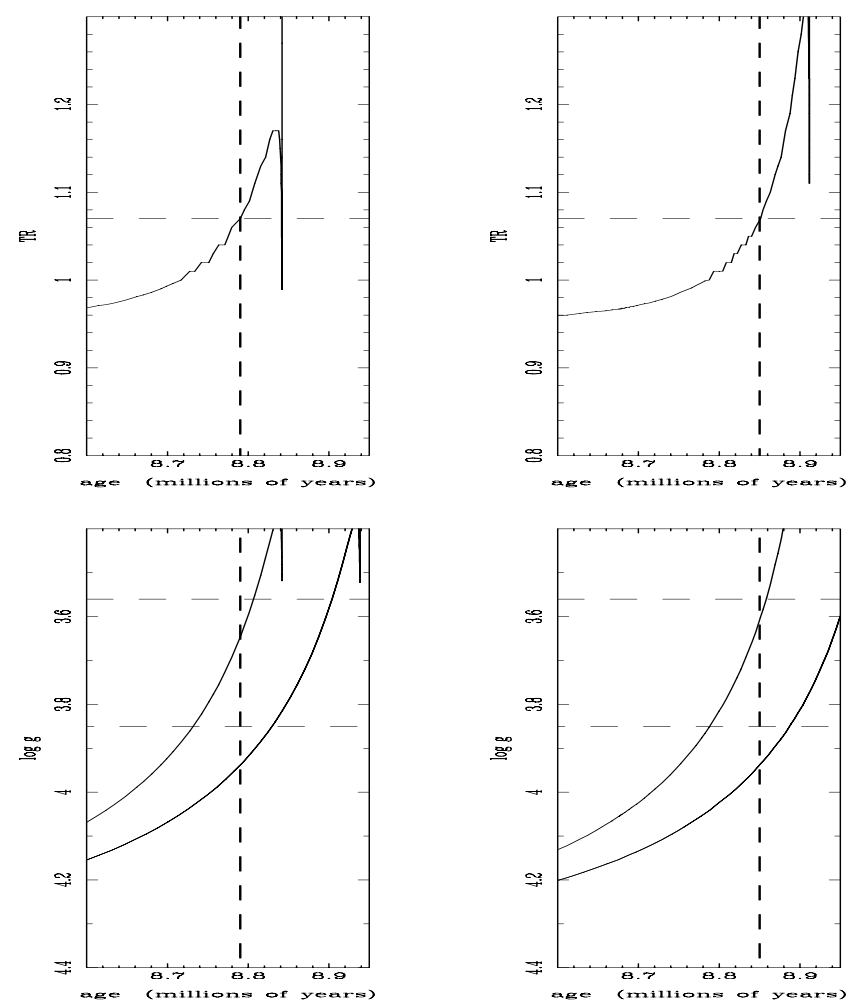

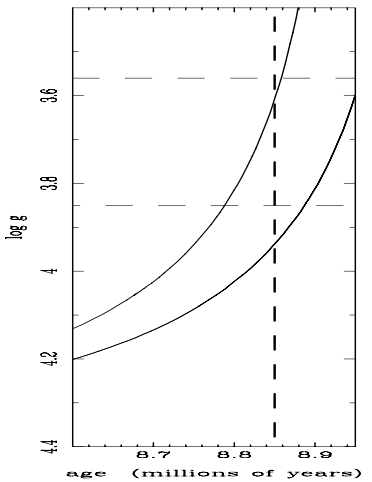

Fig. 6. The same as in Fig. 1 but for V1031 Ori. $(X, Z)=(0.71,0.015)$. 
A. Claret: Does convective core overshooting depend on stellar mass?, Online Material p 5

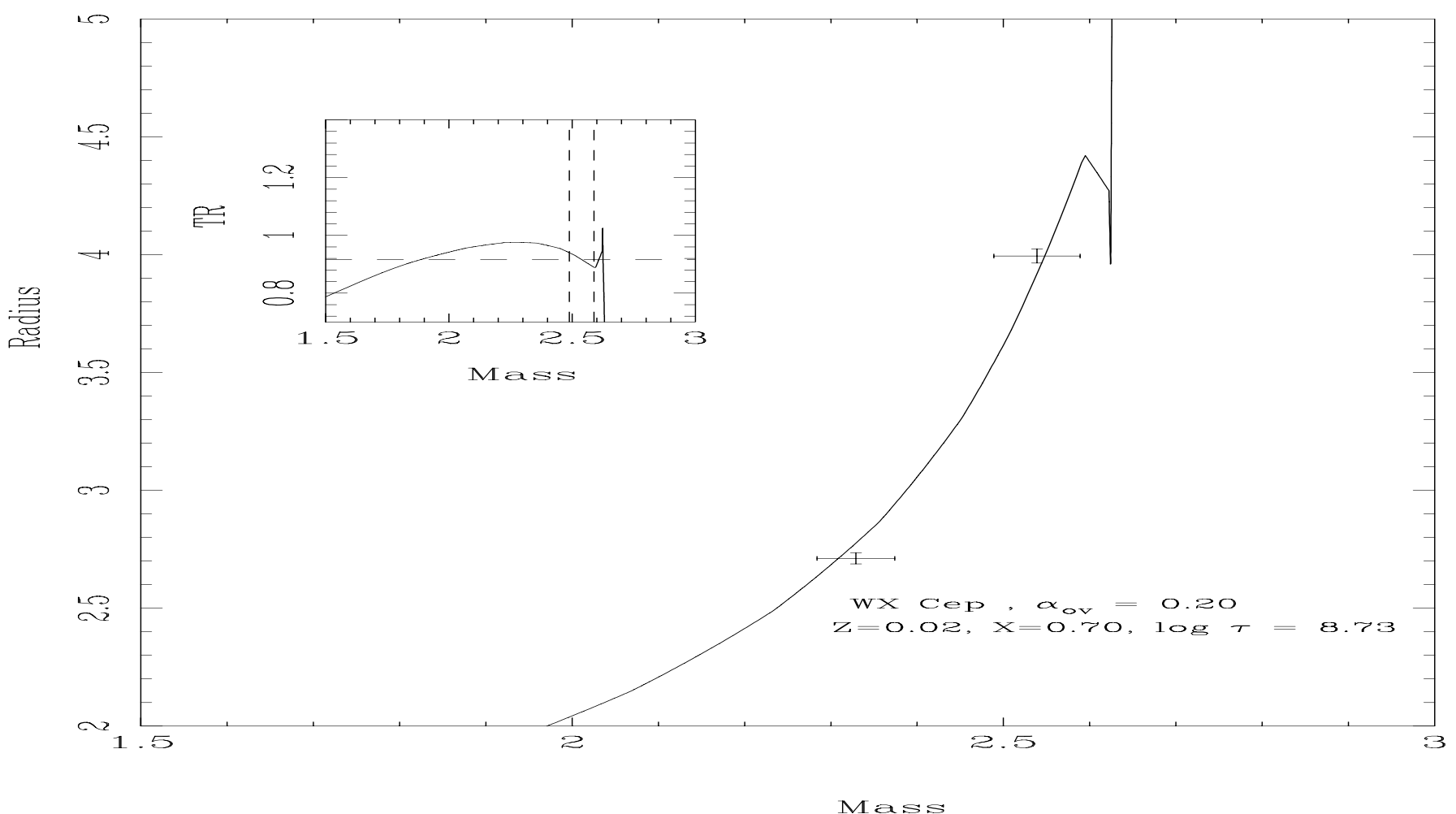

Fig. 7. The same as in Fig. 4 but for WX Cep. Only the case with $\alpha_{\mathrm{ov}}=0.2$ is shown. $(X, Z)=(0.70,0.02)$.

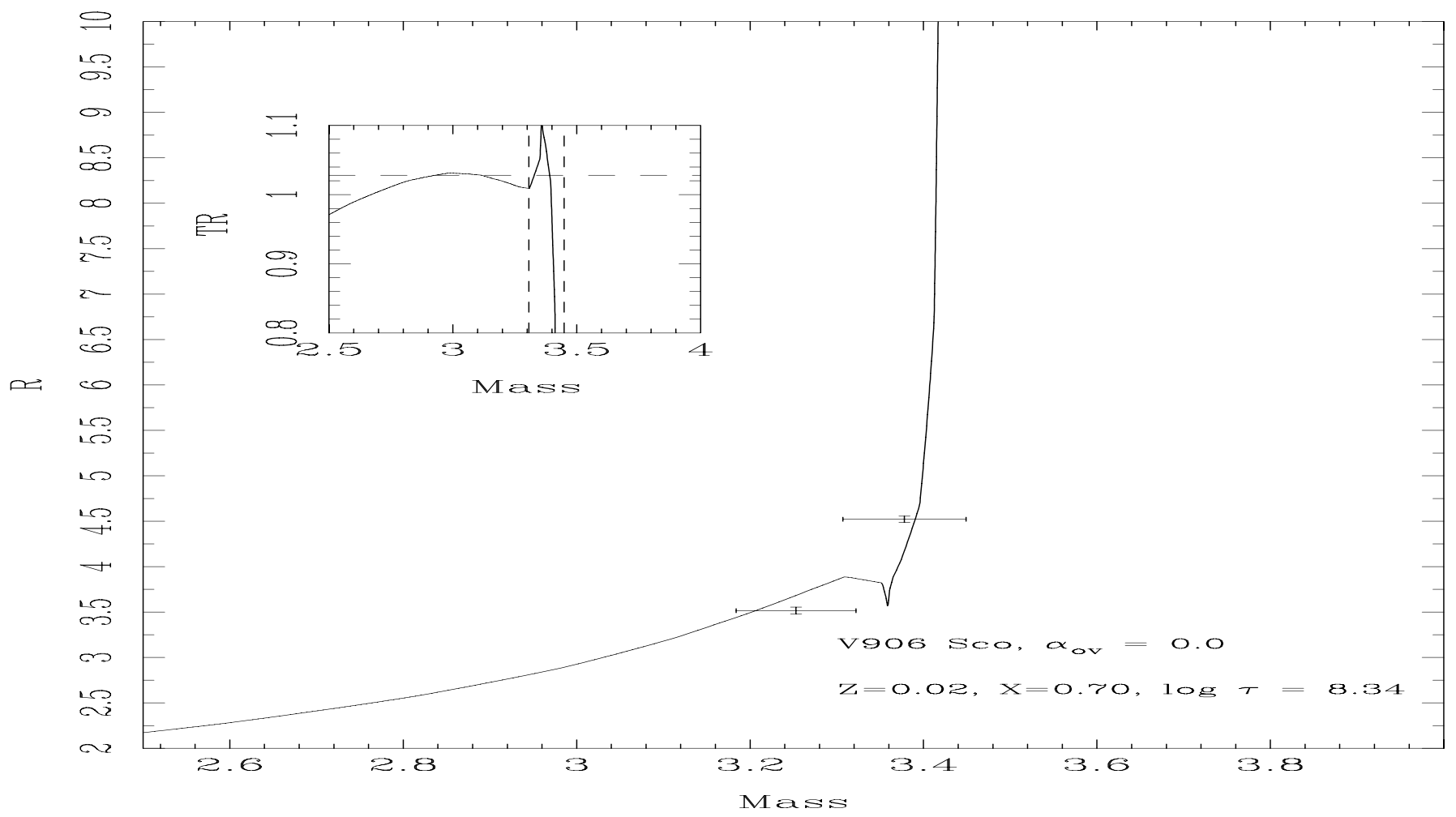

Fig. 8. The same as in Fig. 4 but for V906 Sco. Only the case of no overshooting is shown. $(X, Z)=(0.70,0.02)$. 
A. Claret: Does convective core overshooting depend on stellar mass?, Online Material p 6
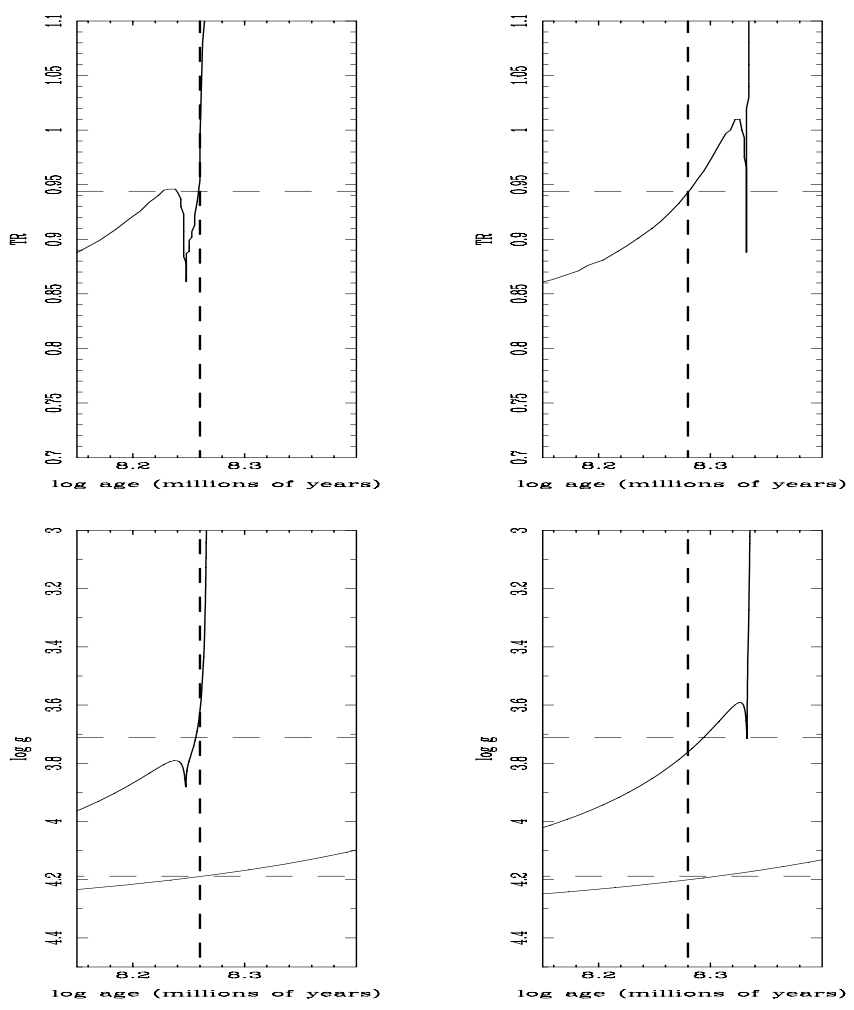

Fig. 9. The same as in Fig. 1 but for $\chi^{2}$ Hyla. $(X, Z)=(0.71,0.015)$.
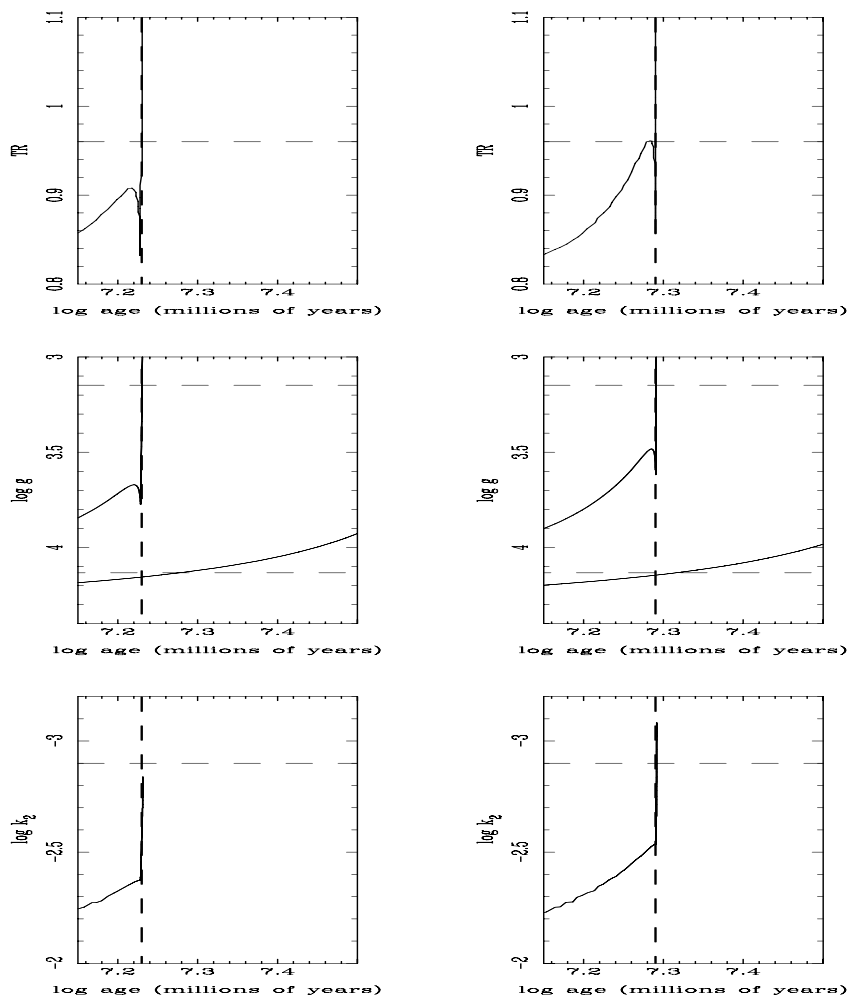
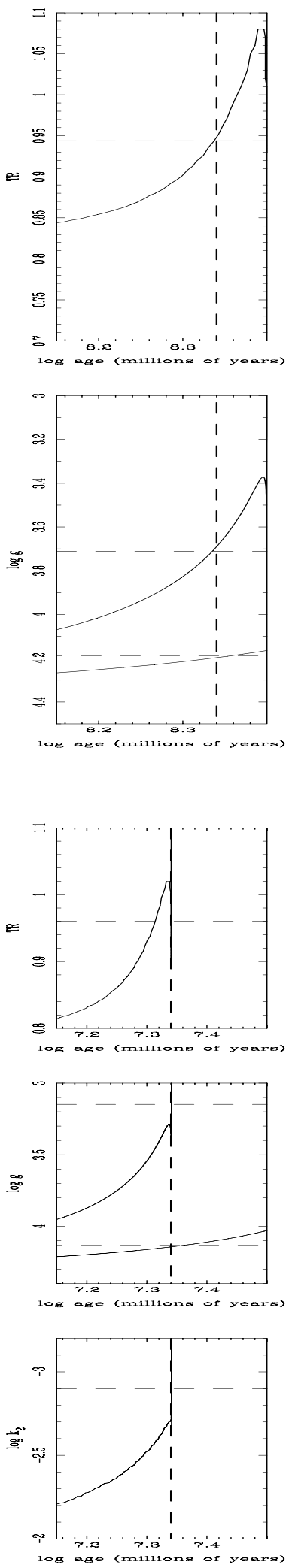
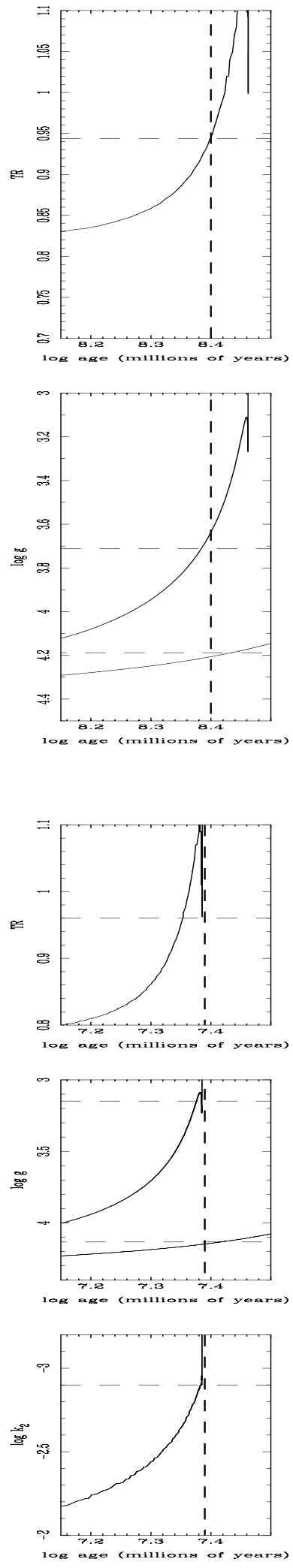

Fig. 10. The same as in Fig. 1 but for V380 Chg. $(X, Z)=(0.71,0.015)$. 
A. Claret: Does convective core overshooting depend on stellar mass?, Online Material p 7
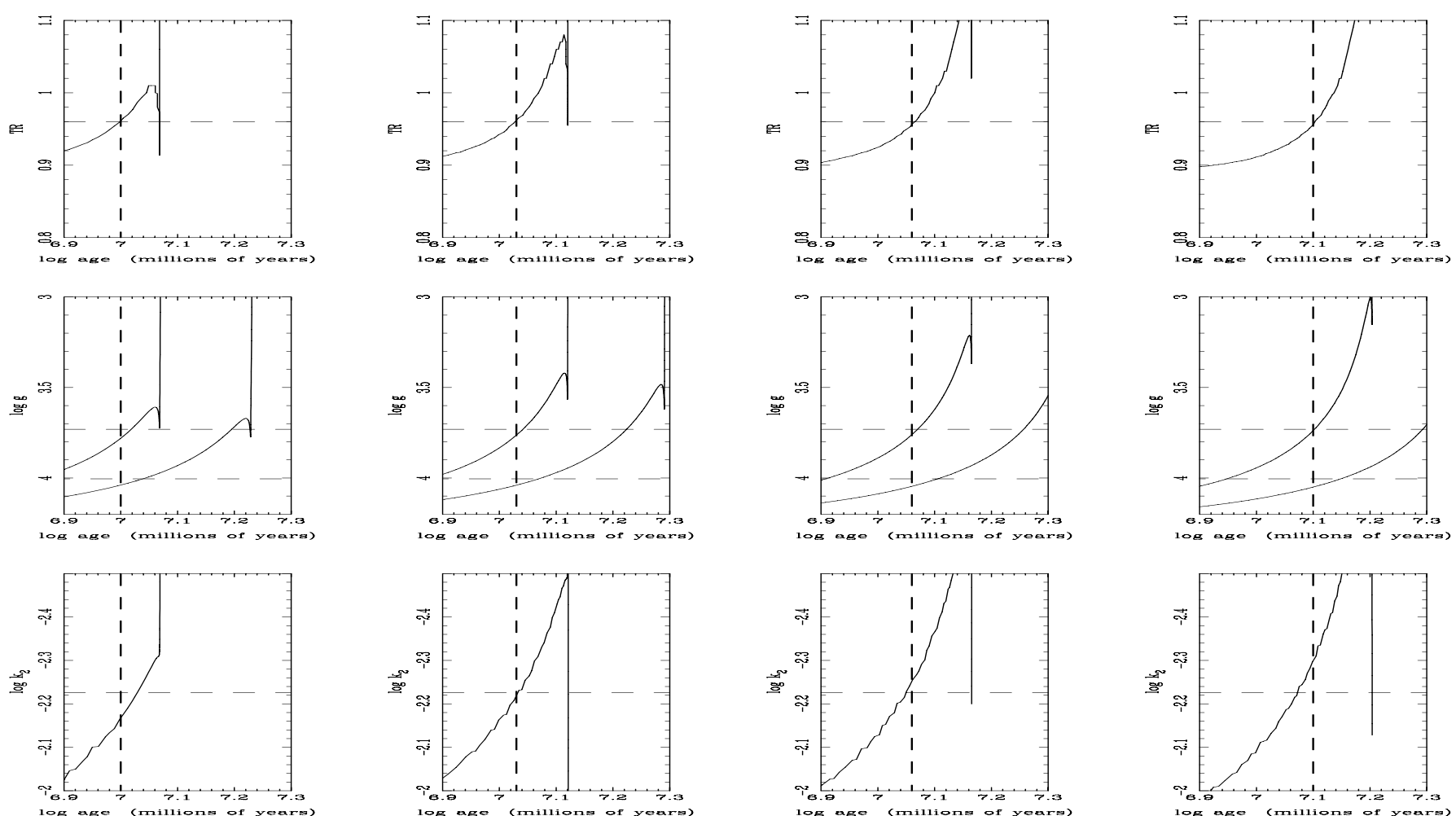

Fig. 11. The same as in Fig. 1 but for V453 Cyg. $(X, Z)=(0.71,0.015)$.

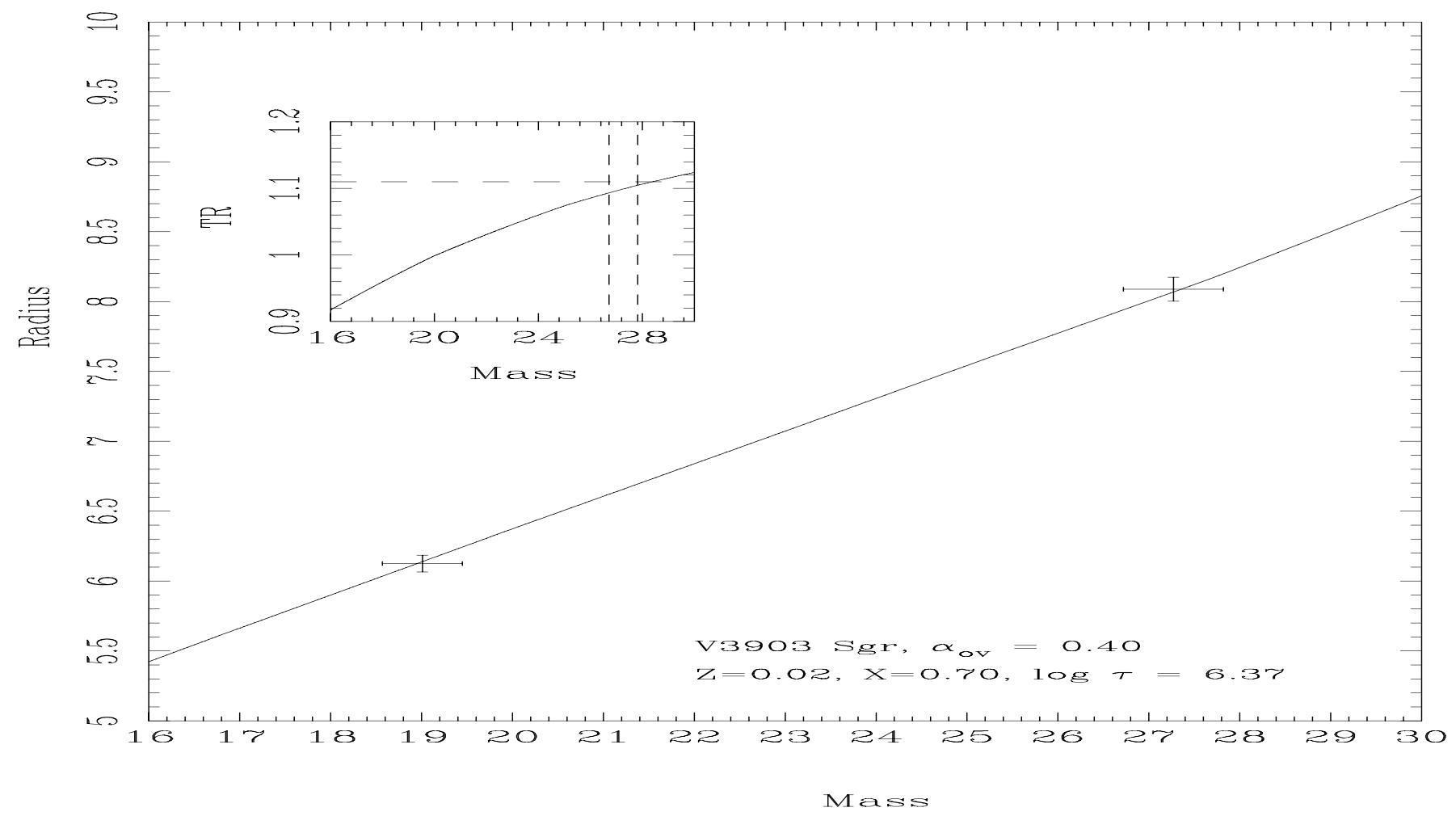

Fig. 12. The same as in Fig. 4 but for V3903 Sgr. Only models with $\alpha_{\mathrm{ov}}=0.4$ are shown. $(X, Z)=(0.70,0.02)$. 